Check for updates

\section{EFAST TRACK}

${ }^{1}$ Department of International Health, Johns Hopkins

Bloomberg School of Public Health, Baltimore, MD, USA

${ }^{2}$ Innovation+Design Enabling Access (IDEA) Initiative, Johns Hopkins Bloomberg School of Public Health, Baltimore, MD, USA

Correspondence to: A D So anthony.so@jhu.edu

(or @anthony_s0888 on Twitter: ORCID 0000-0001-8908-7250)

Additional material is published online only. To view please visit the journal online.

Cite this as: $B M J$ 2020;371:m4750 http://dx.doi.org/10.1136/bmj.m4750

Accepted: 7 December 2020

\title{
Reserving coronavirus disease 2019 vaccines for global access: cross sectional analysis
}

\author{
Anthony D So, ${ }^{1,2}$ Joshua Woo ${ }^{2}$
}

\begin{abstract}
OBJECTIVE

To analyze the premarket purchase commitments for coronavirus disease 2019 (covid-19) vaccines from leading manufacturers to recipient countries.
\end{abstract}

DESIGN

Cross sectional analysis.

DATA SOURCES

World Health Organization's draft landscape of covid-19 candidate vaccines, along with company disclosures to the US Securities and Exchange Commission, company and foundation press releases, government press releases, and media reports.

\section{ELIGIBILITY CRITERIA AND DATA ANALYSIS}

Premarket purchase commitments for covid-19 vaccines, publicly announced by 15 November 2020 .

\section{MAIN OUTCOME MEASURES}

Premarket purchase commitments for covid-19 vaccine candidates and price per course, vaccine platform, and stage of research and development, as well as procurement agent and recipient country.

RESULTS

As of 15 November 2020, several countries have made premarket purchase commitments totaling 7.48 billion doses, or 3.76 billion courses, of covid-19 vaccines from 13 vaccine manufacturers. Just over half (51\%) of these doses will go to high income countries, which represent $14 \%$ of the world's population. The US has reserved 800 million doses but accounts for a fifth of all covid-19 cases globally (11.02 million cases), whereas Japan, Australia, and Canada have

\section{WHAT IS ALREADY KNOWN ON THIS TOPIC}

Reports have suggested that high income countries have made agreements to purchase coronavirus disease 2019 (covid-19) vaccines before they are introduced to the market, but have left the rest of the world with uncertain access Efforts to coordinate global access through the COVAX Facility have lagged behind these arrangements, still require full financing, and have gone forward without the United States or Russia

\section{WHAT THIS STUDY ADDS}

By the end of 2021 up to $40 \%$ of covid-19 vaccine courses from leading manufacturers might potentially remain for low and middle income countriesless if high income countries scale-up existing purchases, more if these countries share what they have procured

Even if these leading manufacturers were all to succeed in reaching their projected maximum production capacity, nearly a quarter of the world's population would not have access to a vaccine until at least 2022

The uncertainty over global access to covid-19 vaccines traces not only to ongoing clinical testing, but also from the failure of governments and vaccine manufacturers to be more transparent and accountable over these arrangements, from fair pricing to equitable allocation

collectively reserved more than one billion doses but do not account for even $1 \%$ of current global covid-19 cases globally ( 0.45 million cases). If these vaccine candidates were all successfully scaled, the total projected manufacturing capacity would be 5.96 billion courses by the end of 2021 . Up to $40 \%$ (or 2.34 billion) of vaccine courses from these manufacturers might potentially remain for low and middle income countries-less if high income countries exercise scaleup options and more if high income countries share what they have procured. Prices for these vaccines vary by more than 10 -fold, from $\$ 6.00$ (£4.50; €4.90) per course to as high as $\$ 74$ per course. With broad country participation apart from the US and Russia, the COVAX Facility-the vaccines pillar of the World Health Organization's Access to COVID-19 Tools (ACT) Accelerator-has secured at least 500 million doses, or 250 million courses, and financing for half of the targeted two billion doses by the end of 2021 in efforts to support globally coordinated access to covid-19 vaccines.

\section{CONCLUSIONS}

This study provides an overview of how high income countries have secured future supplies of covid-19 vaccines but that access for the rest of the world is uncertain. Governments and manufacturers might provide much needed assurances for equitable allocation of covid-19 vaccines through greater transparency and accountability over these arrangements.

\section{Introduction}

With more than 54 million cases and more than a million deaths from coronavirus disease 2019 (covid-19) worldwide, global efforts to produce covid-19 vaccines have gained momentum. ${ }^{1}$ In research and development, 48 candidate vaccines are currently undergoing clinical testing, and at least another 164 candidates are at preclinical stages. ${ }^{2}$ Even with the unprecedented levels of public financing and the accelerated pace of bringing such vaccines to market, global demand will vastly outstrip available supply during this scale-up period. Intense interest has focused on which countries and when populations will have access to safe and effective vaccine candidates emerging from research and development. Pfizer/ BioNTech produced the first covid-19 vaccine to receive regulatory approval, securing emergency use authorization in the United Kingdom in early December 2020. Both Pfizer/BioNTech, and Moderna, with its messenger RNA (mRNA) vaccine, are currently pursuing emergency use authorization from the US Food and Drug Administration.

The potential sources of inequitable and unjust allocation of covid-19 vaccines are not hard to find; 
the solution is considerably more complex. Several countries have prioritized securing vaccine doses to cover their own populations first, even when the need to respond to covid-19 might be greater elsewhere. Facing uncertainty over which vaccines will prove optimally effective, countries with the means to secure future vaccine supplies might ensure against these risks by buying more vaccines than they eventually need or can use. Financing for covid-19 vaccines for low and middle income countries has lagged behind vaccine deals done by high income countries. Different proposed paths to achieving a global equitable allocation of vaccines also exist: the World Health Organization's equitable allocation approach distributes vaccines to reach a percentage of the world's population, whereas other models such as Fair Priority emphasize a needs based metric. ${ }^{34}$ To varying degrees, trade with and travel to countries might face continued disruption until access to effective preventive or treatment measures, such as covid-19 vaccines, becomes more widely available.

High and upper middle income countries with the means for investing in research and development and procuring these future vaccines provide important financing to bring these vaccines to market but could leave others short of such life saving commodities. Bilateral agreements reserving premarket purchase commitments to vaccines not yet on the market, already have been made. Serving as the vaccines pillar of the World Health Organization's Access to COVID-19 Tools (ACT) Accelerator, the COVAX Facility seeks to ensure a more equitable distribution of covid-19 vaccines. It has taken shape with the stewardship of Gavi, the Vaccine Alliance; the Coalition for Epidemic Preparedness Innovations; WHO; and other partners. By pooling resources and candidate vaccines, COVAX provides access to a diversified pool of potential vaccines and affords economies of scale in procuring the most promising ones. ${ }^{5}$ Advance market commitments, cofinanced for eligible countries, are intended to cover initially $3 \%$ and later $20 \%$ of the populations of all countries participating in COVAX. Although open to all countries, COVAX importantly offers a vehicle to finance covid-19 vaccine doses for 92 low income and low-middle income countries through support from the international community. The remainder of the countries that have expressed interest in participating in COVAX would be self-financing, high income and upper middle income countries, currently numbering 97 participants. The US and Russia have thus far opted not to participate in COVAX. ${ }^{6}$

COVAX's goal is to supply at least two billion doses of covid-19 vaccines by the end of 2021, and to date more than $\$ 2.00 \mathrm{bn}$ (€1.49bn; €1.65bn) has been mobilized to support the Gavi COVAX Advance Market Commitment (AMC), enough to reserve one billion doses for AMC eligible countries. ${ }^{7}$ However, at least $\$ 5$ bn more will be needed to procure doses in 2021 as new vaccines become available to the COVAX portfolio. The shortfall in financing a globally coordinated, covid-19 vaccine response makes all the more important the transparency of bilateral agreements between manufacturers and various procurement agents acting on behalf of an individual country or blocs of countries. Countries and COVAX have not published these contracts with vaccine manufacturers, and those secured as part of disclosures to the US Securities and Exchange Commission and under the Freedom of Information Act requests are heavily redacted. ${ }^{8}$ Such limited transparency will fuel concerns about vaccine nationalism, and planning and accountability for ensuring broader access to covid-19 vaccines could be seriously encumbered. ${ }^{9}$ To help lift this nontransparency and to inform equitable allocation of these products, we analyzed the premarket purchase commitments of covid-19 vaccines from leading vaccine manufacturers to countries.

\section{Methods}

Using publicly available information in English up to 15 November 2020, we provide a cross sectional analysis of which countries have reserved covid-19 vaccines, which manufacturers have committed to supply these vaccines, and the potential destination of the vaccine doses. The study focuses on premarket purchase commitments as part of deals or contracts for covid-19 vaccines made before regulatory approval of these products based on completion of a phase III clinical trial. Among these were licensing agreements between the Serum Institute of India and both AstraZeneca/ Oxford University and Novavax for producing a billion doses each of the vaccines.

\section{Search strategy and selection criteria}

Through the WHOdraft landscape of covid-19 candidate vaccines, last updated on 12 November 2020, we identified all companies developing covid-19 vaccines in clinical trials $(n=48)$, and included in this study those companies comprising the sources of premarket purchase commitments to date. ${ }^{2}$ Press releases and US Securities and Exchange Commission disclosures from these companies were used to provide details on the volume of premarket purchase commitments, and the WHO draft landscape of covid-19 candidate vaccines was used to identify the vaccine platform and stage of research and development. A chain sampling approach was then used to include premarket purchase commitments signed by governments and other procurement entities referenced in these company press releases and Securities and Exchange Commission disclosures. Data such as pricing information were then supplemented with details from funding sources (eg, US Biomedical Advanced Reseach and Development Authority and the Coalition for Epidemic Preparedness Innovations) and intergovernmental agency websites as well as media reports. The vaccine platform and stage of research and development were verified against data in the New York Times Coronavirus Vaccine Tracker and Regulatory Affairs Professional Society covid-19 vaccine tracker. ${ }^{10} 11$ When possible, all data were confirmed with multiple sources (see tables in supplemental file). 


\section{Data analysis}

The flows of these vaccine courses are captured using a Sankey diagram, a data visualization commonly used in material flow analysis. The Sankey diagram can show the flows of vaccines from countries or blocs of countries, procured from manufacturers and destined to supply recipient countries or regions. The quantity or magnitude-representing the number of vaccine doses-is depicted by the width of the flow. The Sankey diagrams were visualized using Flourish, an online interactive data visualization software platform (www. IgniteTheIDEA.org/covid-reservedvaccines).

\section{Quality of evidence evaluation}

We excluded deals when the number of vaccine courses was not known. For example, Gamaleya Research Institute has reportedly fielded requests for premarket purchase commitments totaling 1.2 billion doses of its Sputnik vaccine; however, most of these commitments are not publicly disclosed. ${ }^{12}$ Few inconsistencies were found for premarket commitment purchase details across sources, and all were resolved. For example, differences found between sources over the size of premarket purchase commitments traced to media reports that combined, rather than carefully separated, actual premarket purchase commitments and the option to scale-up the volume of such buys later. Other differences in reported premarket purchase commitments resulted from updates to the original agreement. All of the covid-19 vaccine candidates, except SinoVac's, require two doses per course, with Johnson \& Johnson/Janssen's vaccine candidate being tested for courses of both one dose and two doses.

\section{Patient and public involvement}

Prior to the drafting of the manuscript, we reached out to academic colleagues, country and intergovernmental agency policy makers, and civil society for feedback on the value of conducting such an analysis and of using the Sankey diagram as a data visualization tool to convey the study's findings.

\section{Results}

Vaccine prepurchase commitments

Of the 48 covid-19 vaccine candidates undergoing clinical evaluation, 13 manufacturers have entered into premarket purchase commitments for at least 7.48 billion doses, or 3.76 billion courses. High income countries, including the European Union bloc, have reserved $51 \%$ (3851200000 of 7481100000 ) of these doses, or around 3.85 billion doses (table 1 ), though they comprise only 13.7\% (1050756507 of $7674000000)$ of the world's population. Of the 13 manufacturers, only six have sold to low and middle income countries. Of the doses going to low and middle income countries, the majority have been provided by AstraZeneca/Oxford University (2.03 billion doses); Novavax (1.1 billion doses); the Russian firm, Gamaleya Research Institute (349 million doses); and the Chinese firms, SinoVac and CanSino (135 million doses). The covid-19 situation varies markedly across these countries. For example, as of 15 November 2020, the US had reserved 800 million doses but accounted for a fifth of all covid-19 cases globally (11022584 of $54296615)$. By contrast, Japan, Australia, and Canada had reserved more than a billion (1.03 billion) doses, though these three countries combined did not account for even 1\% (445558 of 54296615 ) of all current covid-19 cases (see table S2 in supplemental file).

COVAX has made initial purchases of 300 million vaccine doses from AstraZeneca/Oxford University. ${ }^{13}$ A grant for $\$ 300 m$ from the Bill and Melinda Gates Foundation has secured an additional 200 million doses from either AstraZeneca/Oxford University or Novavax, all with a ceiling price of $\$ 3$ per dose. ${ }^{14}$ This totals to only half of the billion doses for AMC eligible countries, for which financing has been raised, and only a quarter of the at least two billion doses sought by COVAX by the end of 2021. Not included in this count are the 200 million doses of Sanofi/GSK's protein based covid-19 vaccine for which COVAX has also reached an agreement signaling its intention to purchase.

AstraZeneca/Oxford University has reached a licensing agreement with the Serum Institute of India to supply one billion doses for low and middle income countries, with a portion going to India, potentially bolstering the supply to low and middle income countries. The Serum Institute of India has also signed a separate licensing agreement with Novavax for at least an additional one billion doses, also to be distributed to India and other low and middle income countries.

Vaccine manufacturers in Russia and China are also making bilateral agreements with low and middle income countries to supply covid-19 vaccines. While fielding reported requests totaling 1.2 billion doses for its Sputnik V vaccine, the Gamaleya Research Institute has made deals to supply, among other countries, Russia, India, Vietnam, Mexico, and Brazil, with premarket purchase commitments totaling 349 million doses (see fig S1 (Sankey diagram) in supplemental file and the interactive version at www.IgniteTheIDEA. org/covid-reservedvaccines). ${ }^{12}$ With several covid-19 vaccine candidates in phase III clinical trials, China has joined COVAX and also separately reserved vaccine doses from AstraZeneca/Oxford University. China's Fosun Pharmaceutical has also partnered with BioNTech in an effort to bring Pfizer/BioNTech's vaccine candidate to the country. ${ }^{15}$ Similarly, Russia has bought vaccine doses from AstraZeneca/Oxford University.

A second Sankey diagram (see fig S2 in supplemental file and interactive version at www.IgniteTheIDEA.org/ covid-reservedvaccines) shows how various recipient countries have reserved these vaccine candidates at various stages during the research and development process, from preclinical to phase III clinical trials. The US, UK, European Union, Canada, and Japan, among others, have procured vaccine candidates at various stages of research and development. Low and middle income countries, however, have only reserved vaccine candidates in phase III and phase II/III trials. 


\begin{tabular}{|c|c|c|c|c|}
\hline Company & Current R\&D stage & Price pange per course & Platform technology & Total doses committed \\
\hline AstraZeneca/Oxford University & Phase II/III & $\$ 6-\$ 8$ & Non-replicating viral vector & 3221200000 \\
\hline Pfizer/BioNTech & Phase II/III & $\$ 37-\$ 39$ & mRNA & 522400000 \\
\hline CanSino & Phase III & Undisclosed & Non-replicating viral vector & 35000000 \\
\hline Gamaleya Research Institute & Phase III & Undisclosed & Non-replicating viral vector & 349000000 \\
\hline Johnson \& Johnson/Janssen & Phase III & $\$ 20$ & Non-replicating viral vector & 368000000 \\
\hline Medicago & Phase II/III & Undisclosed & Protein subunit & 76000000 \\
\hline Moderna & Phase III & $\$ 30-\$ 74$ & mRNA & 295500000 \\
\hline Novavax & Phase III & $\$ 6-\$ 32$ & Protein subunit & 1626000000 \\
\hline SinoVac & Phase III & Undisclosed & Inactivated & 120000000 \\
\hline CureVac & Phase II & $\$ 24$ & mRNA & 225000000 \\
\hline Sanofi/GSK & Phase I/II & $\$ 42$ & Protein subunit & 532000000 \\
\hline University of Queensland/CSL & Phase I & Undisclosed & Protein subunit & 51000000 \\
\hline Valneva & Preclinical & Undisclosed & Inactivated & 60000000 \\
\hline
\end{tabular}

$\$ 1.00$ (£0.74; €0.82).

$\mathrm{R} \& \mathrm{D}=$ research and development; $\mathrm{mRNA}=$ messenger RNA.

Countries have made premarket purchase commitments for vaccine candidates across a variety of platforms, with 53\% (3.97 billion, 3973200000 of 7481100000 ) concentrated on those based on a non-replicating viral vector platform, 31\% (2.29 billion, 2286000000 of 7481100000$)$ on a protein subunit platform, 14\% (1.04 billion, 1042900000 of 7481100000$)$ on the mRNA platform, and $2 \%$ (180 million, 180000000 of 7481100000$)$ on an inactivated virus platform. Notably, the UK, US, Japan, Canada, and European Union have all reserved vaccine doses across three or more different platforms. However, few low or middle income countries have secured access to vaccines based on the mRNA platform, which is now being released on the market. Pfizer/BioNTech though has begun to strike premarket purchase commitments for its mRNA vaccine with a few upper middle income countries such as Costa Rica and Peru.

The US, UK, European Union, and Canada have placed orders with at least six different manufacturers, Japan with at least four, and Brazil with at least three (see fig S1 in supplemental file and interactive version at www.IgniteTheIDEA.org/covid-reservedvaccines). Among the vaccine manufacturers that have secured such premarket purchase commitments, AstraZeneca/ Oxford University had entered phase III clinical trials ahead of competitors and currently comprises $43 \%$ (3221200000 of 7481100000 ) of all reserved doses to date. AstraZeneca/Oxford University also has provided 56\% (2003000000 of 3629900000 ) of doses reserved for low and middle income countries.

\section{Per capita premarket purchase commitments}

Though multiple elements factor into a country's needs and risk based calculus, the volume of these premarket purchase commitments for covid-19 vaccines can be compared with the population size of the purchasing country or regional bloc and the number of reported covid-19 cases. If these vaccine candidates all successfully scaled, the projected vaccine courses per capita, by country, show that Canada, followed by Australia, the UK, Japan, the
European Union, and US, have reserved at least one vaccine course per person within their respective countries (fig 1). Canada has reserved 9.5 doses, or well over four courses, per person. By contrast, the US has reserved little more than two doses, or one course, per person, and low to middle income countries such as Brazil and Indonesia have reserved less than one course for every two people. As high income countries have locked in vaccine doses, a growing number of premarket purchase commitments have been directed to populations of low and middle income countries. Though earlier deals were agreed bilaterally between vaccine companies and high income countries, recent purchases have focused more on low and middle income countries. For example, over the past few months Russia's Gamaleya Research Institute signed nine deals for premarket purchase commitments-all for low and middle income countries.

\section{Vaccine pricing}

The procurement costs of candidate vaccines currently reflect pandemic pricing, which might not hold (table 1). When tracing the flows from each manufacturer, the two Sankey diagrams (see figs S1 and S2 in supplemental file and the interactive versions at www. IgniteTheIDEA.org/covid-reservedvaccines) yield different insights. The tiered pricing offered by specific vaccine candidates to different countries can be seen in the first Sankey diagram. The pricing of covid-19 vaccine candidates varies widely, even among those using the same platform. AstraZeneca/Oxford University's vaccine candidate is priced at $\$ 8$ per course for countries from the US and UK to Brazil and China, but only $\$ 6$ per course for doses manufactured by the Serum Institute of India and destined for India and other low and middle income countries. Other potential vaccines are several fold more expensive, from BioNTech/Pfizer's vaccine candidate priced at $\$ 39$ per course to Moderna's vaccine candidate priced at \$64-\$74 per course. Vaccine candidates using the same platforms can also vary considerably in price (see fig S2 in supplemental file and interactive version at www.IgniteTheIDEA.org/covid-reservedvaccines). 


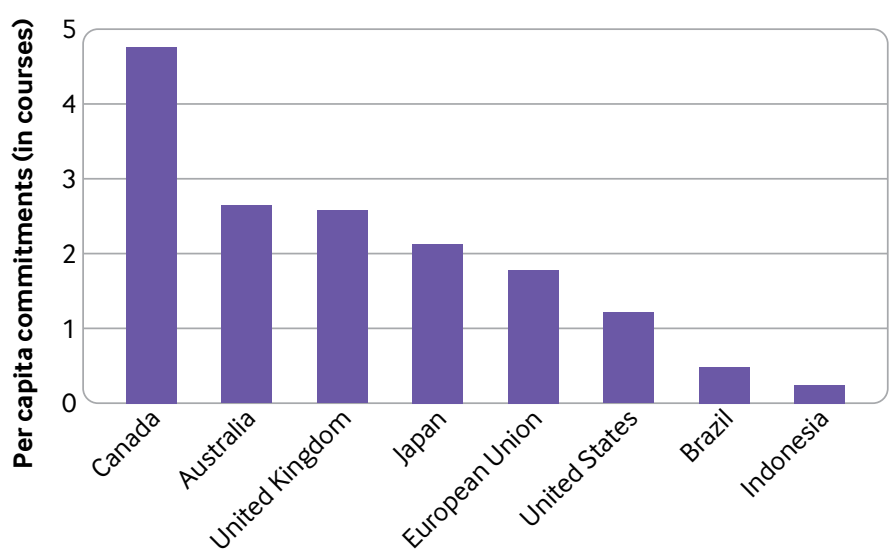

Fig 1 | Premarket commitments for coronavirus disease 2019 vaccines, per capita. Vaccine courses are assumed to require two doses, except for CanSino, which proposes to be one dose per course. Johnson \& Johnson/Janssen's vaccine candidate is being tested for both a one dose and two doses course, but for purposes of this study's analysis, is assumed to be a two dose course vaccine. The vaccine courses for the European Union include premarket purchase commitments not only by the European Union but also by the European Inclusive Vaccines Alliance

Vaccines deploying a non-replicating viral vector range from $\$ 6$ to $\$ 20$ per course, and those using the mRNA platform range from $\$ 24$ to $\$ 74$ per course. Some prices remain undisclosed, and we did not locate information disclosed about the actual production costs for these vaccines.

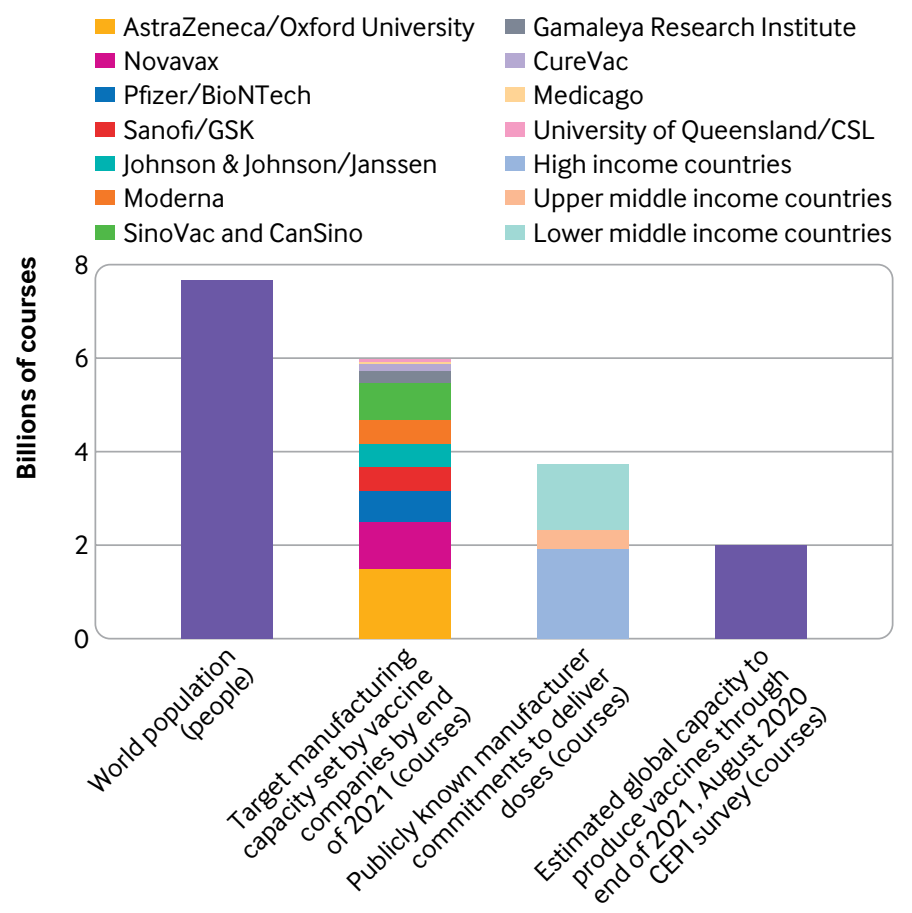

Fig 2 | Projected manufacturing capacity by end of 2021 of lead companies producing coronavirus disease 2019 vaccines. Vaccine courses are assumed to require two doses, except for CanSino, which proposes to be one dose per course. Johnson \& Johnson/ Janssen's vaccine candidate is being tested for both a one dose and two doses course, but for purposes of this study's analysis, is assumed to be a two dose course vaccine. CEPI=Coalition for Epidemic Preparedness Innovations

\section{Vaccine manufacturing capacity}

The publicly known reservations from leading vaccine manufacturers already top 3.76 billion courses, and the anticipated target manufacturing capacity of these companies by the end of 2021 would be nearly 5.96 billion courses (fig 2). This number includes projected vaccine production capacity in China for 610 million doses in 2020 and at least one billion doses annually by the end of $2021 .{ }^{16}$ The cumulative, target manufacturing capacity set by vaccine companies exceeds the Coalition for Epidemic Preparedness Innovations' earlier estimate of the global capacity to produce 2-4 billion doses between October 2020 and the end of 2021. ${ }^{17}$ Not accounting for possible failures of vaccine candidates, financing shortfalls, or logistical challenges, the global manufacturing capacity to deliver covid-19 vaccines by the end of 2021 through the 13 vaccine manufacturers would fall substantially short of covering the world's population of more than 7.67 billion.

Figure 3 depicts the projected manufacturing capacity of all 13 companies with premarket purchase commitments, as well as the number of doses reserved in premarket purchase commitments already made by that company. AstraZeneca/Oxford University-an early candidate into clinical trials-appears to have made public reservations that will account for the entirety of its projected manufacturing capacity of three billion doses through the end of next year (see table S4 in supplemental file). Other companies, however, might have the capacity to make further upfront commitments. In fact, at least 40\% (2337550000 of $5955000000)$ of vaccine courses from these other manufacturers might remain available for low and middle income countries. Under these arrangements, it is not known whether higher income countries might exercise scale-up options beyond their existing premarket purchase commitments or whether high income countries might share what they have procured.

\section{Discussion}

As the first covid-19 vaccine candidates complete clinical testing and reach the market, policy makers and the public have begun to grapple with who will be vaccinated first, when these doses will become available, and where these will initially be targeted. Premarket purchase commitments offer a snapshot of who will probably control covid-19 vaccine doses rolling off production lines. High income countries have reserved just over half of these vaccine doses from 13 leading vaccine manufacturers. Low and middle income countries have the remainder, despite these countries comprising more than $85 \%$ of the world's population. Even if all 13 of these vaccine manufacturers were to succeed in reaching their maximum production capacity, at least a fifth of the world's population would not have access to vaccines until 2022.

High income countries have also taken no chances and secured a broader range of vaccine candidates and platforms. Still, more than $40 \%$ of these 


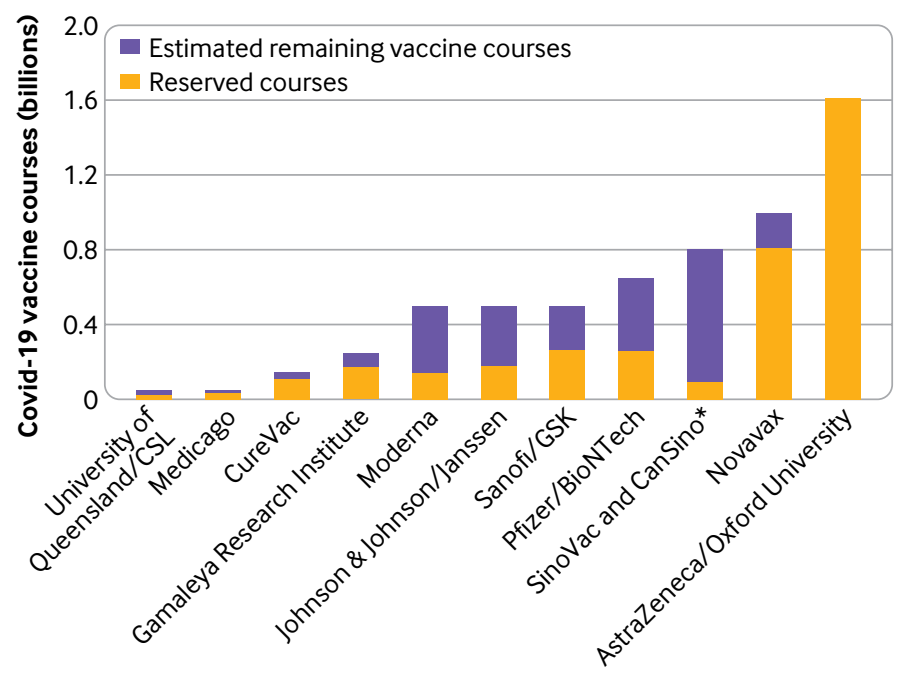

Fig 3 | Estimated remaining courses of coronavirus disease 2019 (covid-19) vaccines. The number of reserved covid-19 vaccine courses is estimated fom the sum total of publicly known, premarket purchase commitments made by that vaccine manufacturer. The remaining covid-19 vaccine courses are estimated against the target manufacturing capacity set by vaccine companies by the end of 2021 . *The projected manufacturing capacity for both SinoVac and CanSino are combined here to represent China's overall publicly disclosed manufacturing capacity of at least one billion doses. Individual manufacturing capacities for SinoVac and CanSino are not known publicly

premarket vaccine commitments for high income countries depend on the success of one candidate, the AstraZeneca/Oxford University vaccine. This vaccine candidate not only has the lowest price, but also only requires traditional refrigeration for transport and storage. AstraZeneca/Oxford University has made the largest premarket purchase commitment to low and middle income countries. By contrast, only high and upper middle income countries have been able to procure mRNA vaccines-notably from Pfizer/BioNTech and Moderna. While these particular vaccines might be among the first to obtain regulatory approval, they require a cold chain infrastructure not readily available in resource limited settings.

\section{Policy implications}

The COVAX Facility could play a key role in ensuring access to covid-19 vaccines. However, its target of two billion doses by the end of 2021 is still short on premarket vaccine commitments and financing to deliver on this goal. Several countries have contributed to COVAX to support AMC eligible participants for access to covid-19 vaccines. On behalf of COVAX, Unicef and the Pan American Health Organization's Revolving Fund for Access to Vaccines have launched a tender for all developers of covid-19 vaccines to submit proposals to help meet this demand in the coming year. Among the 13 vaccine manufacturers with premarket purchase commitments, most might have potential doses not yet reserved. On the supply side, 35 of the 48 vaccine manufacturers now in clinical testing also do not have publicly known, premarket purchase commitments, but without such existing commitments, it is also not clear whether they have the financing or facilities to scale. On the demand side, COVAX might require copayments of $\$ 1.60$ per dose for upfront, committed purchases by self-financing countries. ${ }^{18}$ Such copayments might compel some countries to secure external loans or financing, perhaps from the World Bank's \$12bn envelope for financing covid-19 vaccines, treatments, and tests. So the existing 10fold variation in vaccine prices could pose serious challenges to affordable access.

Several analyses have highlighted the number of vaccine doses reserved by high income countries as a sign of vaccine nationalism. ${ }^{19-21}$ This study goes further in unpacking whether there are potential reasons that explain why some countries have purchased more vaccine courses. Several reasons to explore include providing upfront financing to ensure promising covid-19 vaccine candidates come to market and can be scaled, ensuring against the risk of failure of covid-19 vaccines not yet approved for use, and stockpiling for distribution or donation to other countries.

All of the vaccine candidates in this study have received premarket purchase commitments, but some were also supported by funding for research and development. For example, Moderna has received \$955m in Biomedical Advanced Research and Development funding for research and development, and a premarket purchase commitment of $\$ 1.53 \mathrm{bn}$, whereas Pfizer/BioNTech has benefited from $€ 375 \mathrm{~m}$ from the German Federal Ministry of Education and Research and another $€ 100 \mathrm{~m}$ in debt refinancing from the European Investment Bank. ${ }^{22} 23$ However, these monies have largely funded bilateral agreements between high income countries and specific vaccine manufacturers, and at this stage no public commitments to sharing these reserved vaccine doses with low and middle income countries can be documented. These bilateral agreements have prompted concerns that they might have also bidded up the price of vaccines.

The number of covid-19 vaccine courses per capita is one measure of how countries ensure against the risk of potential failure of vaccine candidates. The number of courses showed considerable variability across high income countries, from over four courses per person in Canada to just over one course in the US. Current needs, as reflected in differences in covid-19 burden, did not explain this variability. A fifth of the world's covid-19 cases have occurred in the US, whereas Canada, Japan, and Australia combined do not even comprise 1\% of this total. Anticipated vaccine hesitancy also does not seem to account for these differences. In fact, a recent survey found the public's willingness to take a safe and effective covid-19 vaccine is slightly lower in Canada than in the US. ${ }^{24}$ Reportedly, the US agreement with Moderna includes an option for scale-up. Though these contracts are not fully transparent, scale-up options could potentially explain why the US might not require as sizeable, premarket purchase commitments. Still the doses secured by low and middle income countries, such as Brazil and Indonesia, remain considerably less. It remains unknown how many countries will adhere to the stepwise, population 
based distribution of vaccines, proposed under the WHO's equitable allocation scheme, or whether some might double dip, sourcing vaccine supplies both from bilateral agreements and from COVAX.

Without a globally coordinated approach to distributing covid-19 vaccines, stockpiling supplies might result. Importantly, with financing and logistical challenges in delivery of these vaccines, stockpiles might not be so easily shared. Uncertainty over which vaccines would succeed or fail, the time it has required to pull together participation and financing of COVAX, and the bilateral agreements that had been struck within that timeframe have also contributed to this situation. COVAX is stepping up such global coordination. The US and Russia, however, have opted not to participate. Yet these two countries are home to key vaccine companies responsible for a major proportion of the globe's covid-19 vaccine manufacturing capacity. Uncertainty over the effectiveness, duration of immunity, safety, and dosing regimens of vaccine candidates will continue to diminish as clinical testing is completed.

The first covid-19 vaccine to market might not be the most effective nor the best suited for reaching or immunizing all populations. This will lead to a situation whereby competing vaccines reaching the market might differ considerably in effectiveness in different country settings. Second generation vaccines might be more effective but face additional challenges: scale-up facilities locked into existing vaccine production; switching costs to transition to more effective vaccines; vaccine hesitancy heightened by any adverse reactions from the first generation of vaccines; and likely barriers to intellectual property rights as patent thickets emerge. This makes critical for scale-up the global and country level coordination of head-to-head trials among leading vaccine candidates, the emergence of second generation vaccines that outperform those first to market, and the ability to switch manufacturing facilities from less effective vaccines to more optimal vaccines.

\section{Strengths and limitations of this study}

This study has several limitations. First, neither governments nor vaccine manufacturers are required to disclose publicly contracts containing premarket purchase commitments. Public awareness of sizable purchase commitments, however, benefits the vaccine manufacturer seeking financial investors and manufacturing facilities to scale production, as well as governments seeking to reassure the public. Second, contractual arrangements disclosed to the public have been heavily redacted. ${ }^{8}$ This has left uncertainty over who has priority among those making premarket purchase commitments. However, the norms governing pooled procurement efforts such as COVAX, the geographical siting of scale-up manufacturing facilities, and control over production inputs such as glass vials as well as the local infrastructure for vaccine uptake will help determine who will be first in line for covid-19 vaccines. Third, we could not determine whether vaccine manufacturers could feasibly attain their projected manufacturing capacity by the end of 2021. This will depend on the uncertain timeline to regulatory approval, the availability of financing for scale-up, and the competition narrowing the field of viable vaccine candidates. Moreover, this study only assessed the potential production capacity of 13 vaccine manufacturers that have made publicly known, premarket purchase commitments. The projected manufacturing capacity by the end of 2021 are based on publicly available, company based estimates assuming successful regulatory approval, scale-up, and demand for the company's vaccine candidate. However, these are likely to be the companies most well positioned to scale-up first with public sector backing.

Still this study reveals a portrait, in part, of how high income countries have sought to secure future supplies of covid-19 vaccines, but have left much of the rest of the world with uncertain access. Those hopes are today focused on a handful of lead vaccine candidates, some of which might yet falter or fail. Greater transparency is needed about manufacturer agreements as well as the underlying costs of research and development, public sector financing for covid-19 vaccine candidates, and pricing arrangements. Pooled procurement, coordinated production, and head-tohead trials of covid-19 vaccines will require global collective action. Ensuring an effective response to this pandemic will require more-the commitment of high income countries to share in an equitable distribution of covid-19 vaccines across the world.

We thank for their support the Innovation+Design Enabling Access Initiative, Johns Hopkins Bloomberg School of Public Health; the Johns Hopkins Alliance for a Healthier World; and the Open Society Foundation. Data used in this study are provided in the supplemental file.

The table and figures are also available at https://www.ignitetheidea. org/covid-reservedvaccines. The Sankey diagrams (see also supplementary figures S1 and S2) on the website are interactive data visualizations.

Contributors: ADS conceived and designed the study. ADS and JW collected the data. JW visualized the data with feedback from ADS. ADS took the lead in writing the manuscript, but JW also actively participated in analyzing the data and writing and revising the manuscript. Both authors have read and agreed to the published version of the manuscript, and take responsibility as guarantors of its content. The corresponding author attests that all listed authors meet authorship criteria and that no others meeting the criteria have been omitted.

Funding: This study was supported by the Innovation+Design Enabling Access Initiative at the Johns Hopkins Bloomberg School of Public Health; the Johns Hopkins Alliance for a Healthier World; and the Open Society Foundation (grant OR2017-38241). JW was supported under the Johns Hopkins Alliance for a Healthier World. The funders had no role in considering the study design or in the collection, analysis, interpretation of data, writing of the report, or decision to submit the article for publication.

Competing interests: All authors have completed the ICMJE uniform disclosure form at www.icmje.org/coi_disclosure.pdf and declare: ADS received support from the Innovation+Design Enabling Access Initiative at the Johns Hopkins Bloomberg School of Public Health; the Johns Hopkins Alliance for a Healthier World; and the Open Society Foundation. JW was supported as a Global Health Equity Scholar under the Johns Hopkins Alliance for a Healthier World. Outside of the submitted work, both authors have provided unpaid advisory input to the Pan American Health Organization's Revolving Fund for Access to Vaccines and have previously received grants for unrelated work from the World Health Organization within the past three years. 
Ethical approval: Not required.

Data sharing: No additional data available.

The authors (ADS and JW) affirm that the manuscript is an honest, accurate, and transparent account of the study being reported; that no important aspects of the study have been omitted; and that any discrepancies from the study as planned (and, if relevant, registered) have been explained.

Dissemination to participants and related patient and public communities:The authors plan to disseminate these findings to the general public both by press release and through an interactive data visualization made available on the Johns Hopkins Bloomberg School of Public Health's Innovation+Design Enabling Access (IDEA) Initiative website.

Provenance and peer review: Not commissioned; externally peer reviewed.

This is an Open Access article distributed in accordance with the Creative Commons Attribution Non Commercial (CC BY-NC 4.0) license, which permits others to distribute, remix, adapt, build upon this work non-commercially, and license their derivative works on different terms, provided the original work is properly cited and the use is noncommercial. See: http://creativecommons.org/licenses/by-nc/4.0/.

1 Center for Systems Science and Engineering, Johns Hopkins University. COVID-19 Dashboard. [Accessed 15 Nov 2020.]

2 World Health Organization. DRAFT landscape of COVID-19 candidate vaccines, 12 November, 2020. https://www.who.int/publications/m/ item/draft-landscape-of-covid-19-candidate-vaccines.

3 World Health Organization. WHO Concept for fair access and equitable allocation of COVID-19 health products. Final working version 9 September 2020. Geneva, Switzerland: World Health Organization, 2020. https://www.who.int/publications/m/item/ fair-allocation-mechanism-for-covid-19-vaccines-through-the-covaxfacility.

4 Emanuel EJ, Persad G, Kern A, et al. An ethical framework for global vaccine allocation. Science 2020;369:1309-12. doi:10.1126/ science.abe 2803

5 COVAX. Commitment Agreements, September 30, 2020. https:// www.gavi.org/sites/default/files/covid/pr/COVAX_CA_COIP_List_ COVAX_PR_30-09.pdf.

6 Gavi, the Vaccine Alliance. COVAX, the ACT-Accelerator Vaccines Pillar: Insuring Accelerated Vaccine Development and Manufacture, 2020. https://www.gavi.org/sites/default/files/covid/COVAX-Pillarbackground.pdf.

7 Gavi COVAX Facility. Over US\$2 billion raised to support equitable access to COVID vaccines with additional US $\$ 5$ billion needed in 2021. Press release, 13 November 2020. https://www.gavi.org/ news/media-room/over-us-2-billion-raised-support-equitableaccess-covid-vaccines-additional-us-5

8 Mancini DP. Vaccine contracts shrouded in secrecy despite massive public funding. Financial Times, 23 November 2020. https://www. ft.com/content/95c49b5a-f2c7-49a3-9ac5-3e7a66e3ad6b

9 Human Rights Watch. Whoever Finds the Vaccine Must Share It: Strengthening Human Rights and Transparency around COVID-19 Vaccines, October 2020. ISBN: 978-1-62313-8769 https://www. hrw.org/sites/default/files/media_2020/10/globalvaccine1020_ web.pdf

10 Corum |, Wee S, Zimmer C Coronavirus Vaccine Tracker. New York Times. [Accessed 18 September 2020.] https://www.nytimes.com/ interactive/2020/science/coronavirus-vaccine-tracker.html.

11 Craven J. COVID-19 vaccine tracker. Regulatory Affairs Professionals Society. [Accessed 18 September 2020.] https://www.raps.org/newsand-articles/news-articles/2020/3/covid-19-vaccine-tracker.
12 Sputnik V. Second interim analysis of clinical trial data showed a $91.4 \%$ efficacy for the Sputnik V vaccine on day 28 after the first dose; vaccine efficacy is over $95 \% 42$ days after the first dose. Press Release, 24 November 2020. https://sputnikvaccine.com/ newsroom/pressreleases/second-interim-analysis-of-clinical-trialdata-showed-a-91-4-efficacy-for-the-sputnik-v-vaccine-on-d/.

13 AstraZeneca. Agreements with CEPI and Gavi and the Serum Institute of India will bring vaccine to low and middle-income countries and beyond. Press release, 4 June 2020. https://www.astrazeneca.com/ media-centre/press-releases/2020/astrazeneca-takes-next-stepstowards-broad-and-equitable-access-to-oxford-universitys-covid-19vaccine.html.

14 Gavi the Vaccine Alliance. New collaboration makes further 100 million doses of COVID-19 vaccine available to low- and middleincome countries. Press release, 29 September 2020. https://www. gavi.org/news/media-room/new-collaboration-makes-further-100million-doses-covid-19-vaccine-available-low.

15 BioNTech. BioNTech and Fosun Pharma form COVID-19 vaccine strategic alliance in China. Press release, 16 March 2020. https:// investors.biontech.de/news-releases/news-release-details/biontechand-fosun-pharma-form-covid-19-vaccine-strategic.

16 McNeil S. China aims to make 1 billion COVID-19 vaccine doses a year. Associated Press, 25 September 2020. https://www. apnews.com/article/virus-outbreak-beijing-china-archivea9fc0c458fda6a950da5f2cc0f4a354b.

17 Coalition for Epidemic Preparedness Innovations. CEPI COVID-19 Manufacturing Survey Results Analysis. Report, 29 June 2020 https://cepi.net/wp-content/uploads/2020/08/CEPI_Survey-ofglobal-drug-substance-and-drug-product-landscape-June-2020_ RELEASED-1.pdf.

18 Gavi, the Vaccines Alliance. COVAX Explained, 3 September 2020. https://www.gavi.org/vaccineswork/covax-explained.

19 Oxfam International. Small group of rich nations have bought up more than half the future supply of leading COVID-19 vaccine contenders, 17 September 2020. https://www.oxfam.org/en/pressreleases/small-group-rich-nations-have-bought-more-half-futuresupply-leading-covid-19

20 Duke Global Health Innovation Center. Will Low-Income Countries Be Left Behind When COVID-19 Vaccines Arrive? 9 November 2020. https://globalhealth.duke.edu/news/will-low-income-countries-beleft-behind-when-covid-19-vaccines-arrive

21 Global Justice Now. Most of Pfizer's vaccine already promised to richest, campaigners warn. 11 November 2020. https://yubanet. com/world/most-of-pfizers-vaccine-already-promised-to-richestcampaigners-warn/

22 BioNTech press release. BioNTech to Receive up to $€ 375 \mathrm{M}$ in Funding from German Federal Ministry of Education and Research to Support COVID-19 Vaccine Program BNT162, 15 September 2020. https://www.globenewswire.com/newsrelease/2020/09/15/2093535/0/en/BioNTech-to-Receive-up-to$375 \mathrm{M}$-in-Funding-from-German-Federal-Ministry-of-Education-andResearch-to-Support-COVID-19-Vaccine-Program-BNT162.html

23 European Investment Bank. Investment Plan for Europe: European Investment Bank to provide BioNTech with up to $€ 100$ million in debt financing for COVID-19 vaccine development and manufacturing, 11 June 2020. https://ec.europa.eu/commission/presscorner/detail/en/ ip_20_1034

24 Lazarus JV, Ratzan SC, Palayew A, et al. A global survey of potential acceptance of a COVID-19 vaccine. Nat Med 2020, published online first 20 October. doi:10.1038/s41591-020-1124-9

Supplemental information: figures S1 and S2 and tables S1-S4 